\title{
Prolonged decay of molecular rate estimates for metazoan mitochondrial DNA
}

Martyna Molak, Simon Y W Ho

Evolutionary timescales can be estimated from genetic data using the molecular clock, calibrated by fossil or other evidence. However, estimates of molecular rates in mitochondrial DNA appear to scale negatively with the age of the clock calibration. Although such a pattern has been observed in a limited range of data sets, it has not been studied on a large scale in metazoans. In addition, there is uncertainty over the temporal extent of the time-dependent pattern in rate estimates. Here we present a meta-analysis of 239 rate estimates from metazoans, representing a range of timescales and taxonomic groups. We found evidence of time-dependent rates in both coding and non-coding mitochondrial markers, in every group of animals that we studied. The negative relationship between the estimated rate and time persisted across a much wider range of calibration times than previously suggested. This indicates that, over long timeframes, purifying selection gives way to mutational saturation as the main driver of the timedependent biases in rate estimation. The results of our study stress the importance of accounting for time-dependent biases in estimating evolutionary rates using mitochondrial data regardless of the timescale over which they are inferred. 


\section{Prolonged decay of molecular rate estimates for metazoan mitochondrial DNA}

2 Martyna Molak ${ }^{1,2}$, Simon Y.W. Ho ${ }^{1}$

$3 \quad{ }^{1}$ School of Biological Sciences, University of Sydney, Sydney, Australia

$4 \quad{ }^{2}$ Museum and Institute of Zoology, Polish Academy of Sciences, Warsaw, Poland

5 Corresponding author: Martyna Molak, Museum and Institute of Zoology, Polish Academy of

6 Sciences, Wilcza 64, 00-679 Warszawa, Poland; Phone: +48226293221; Email:

7 martyna.molak@gmail.com

8 Keywords: substitution rate, molecular clock, evolutionary timescale, calibration, time-dependent

9 rates 
11 Understanding the tempo and mode of the molecular evolutionary process is one of the

12 fundamental goals of biological research. Determining rates and timescales of evolution allows us

13 to explore such questions as the co-evolution of species (Reed et al., 2007), causes of extinction

14 (Lorenzen et al., 2011), and drivers of diversification (Jetz et al., 2012). Molecular estimates of

15 evolutionary timescales are made using the molecular clock, which assumes that DNA evolves at

16 a constant rate among lineages (Zuckerkandl \& Pauling, 1962). With improving knowledge of the

17 molecular evolutionary process, molecular-clock methods have undergone considerable

18 development over the past five decades (Ho, 2014; Kumar, 2005), with various forms of rate

19 variation being able to be taken into account (Ho \& Duchêne, 2014).

20 To estimate evolutionary rates from DNA sequence data, molecular clocks need to be calibrated.

21 This involves using independent information to constrain the age of one or more nodes in a

22 phylogenetic analysis. There are several types of evidence that can provide calibrations for

23 molecular clocks (for a recent review, see Hipsley \& Müller, 2014). One of the primary sources is

24 the fossil record, which can provide an indication of the first appearance of a lineage and thus

25 place a minimum bound on when it diverged from its sister lineage. The ages of nodes in the tree

26 can also be estimated using biogeographic hypotheses. For example, the rise of barriers to gene

27 flow or new habitats for colonization can provide age estimates for divergences between sister

28 species (e.g., Fleischer et al., 1998).

29 Over short timescales, molecular clocks can be calibrated by heterochronous sampling or when

30 the ancestry of the samples is known through a pedigree. Heterochronous sampling is common in

31 studies of viruses and of ancient DNA. If the ages of the samples are sufficiently distinct from

32 one another, they can be used to calibrate estimates of evolutionary rates (Drummond et al., 2003; 
33 Molak et al., 2013). In pedigree studies, the relationships among samples have been documented,

34 and their divergence times might be known exactly.

35 The most significant challenge to the molecular clock has been widespread evidence of rate

36 variation among lineages. These patterns of rate variation can be partly explained by differences

37 in generation time, longevity, and other life-history characters (Bromham, 2009). Recently,

38 however, there has been growing evidence of heterogeneity in rate estimates across timescales.

39 For example, mitochondrial rates estimated in phylogenetic studies are about an order of

40 magnitude lower than those estimated in analyses of pedigrees (Ho \& Larson, 2006; Howell et

41 al., 2003). More generally, estimated rates appear to be time-dependent, showing a negative

42 relationship with the timescale over which they are measured (Ho et al., 2011; Ho et al., 2005).

43 This pattern has been observed in coding and non-coding mitochondrial DNA from a range of

44 taxa, including humans (Endicott et al., 2009; Henn et al., 2009; Rieux et al., 2014), fish

45 (Burridge et al., 2008; Genner et al., 2007), birds (García-Moreno et al., 2006; Ho et al., 2005),

46 and invertebrates (Crandall et al., 2012; Gratton et al., 2008; Ho \& Lo, 2013; Papadopoulou et al.,

47 2010).

48 Various explanations have been proposed for time-dependent biases in rate estimation. These

49 include natural selection, calibration errors, model misspecification, sequence errors, and biases

50 in phylogenetic estimation and sampling. The first three factors are likely to be the most

51 important (see Ho et al., 2011 for a review). Transient deleterious mutations tend to inflate

52 estimates of rates over short timescales, but they are removed by selection over longer timescales

53 (Duchêne et al., 2014; Soares et al., 2009; Woodhams, 2006). Calibration errors mostly derive

54 from the assumption that the genetic split between lineages coincides with the divergence of

55 populations. The impact of calibration error is more severe in short-term rate estimates, where the

56 discrepancy between genetic and population split time constitutes a large proportion of the 
57 overall timescale (Ho et al., 2005; Peterson \& Masel, 2009). Additionally, estimates of rates can

58 be misled if they are obtained using models that provide a poor reflection of real evolutionary and

59 demographic processes (Emerson, 2007; Navascues \& Emerson, 2009; Soubrier et al., 2012).

60 Misspecified models of nucleotide substitution can lead to underestimates of saturation, causing

61 underestimates of rates over long evolutionary timescales (dos Reis \& Yang, 2013; Duchêne et

62 al., 2014; Ho et al., 2007b).

63 The time-dependent biases in molecular rate estimation have important implications for studies of

64 recent evolutionary timescales. For example, the ages of coalescence events within populations

65 will tend to be overestimated if they are based on long-term rates calibrated using fossil data (Ho

66 et al., 2008). This time-dependence, together with substantial variation in rates among different

67 taxa, also calls into question the application of so-called 'standard' mitochondrial rates, such as

68 those estimated in studies of lizards (Macey et al., 1998), birds (Shields \& Wilson, 1987; Weir \&

69 Schluter, 2008), mammals (Brown et al., 1979), and arthropods (Brower, 1994) and used in many

70 subsequent studies. Time-dependent biases in rate estimation might also contribute to a wider

71 discussion about discrepancies between molecular and palaeontological estimates of divergence

72 times (Benton \& Ayala, 2003).

73 Although there is a clear discrepancy between rates estimated on short and long timescales, there

74 remains uncertainty about how rates scale with time. A major hindrance has been the paucity of

75 reliable calibrations on intermediate timescales. In this regard, ancient DNA data can be

76 particularly useful in bridging the gap between short- and long-term rate estimates (Ho et al.,

77 2007a). Because ages are assigned directly to sequences in the analysis, no assumptions need to

78 be made about the timing of population splits and their correspondence to genetic divergences

79 (Rambaut, 2000). Unfortunately, ancient DNA analyses are constrained by the post-mortem

80 degradation of DNA and the limited reach of radiocarbon dating. 
81 Accurate characterization and quantification of time-dependent biases in rate estimation is crucial

82 for understanding their causes and accounting for their effects. Studies of time-dependence in rate

83 estimates have typically involved limited data sets, comprising small numbers of taxa or

84 sequences. This leaves open questions concerning the prevalence and ubiquity of these biases, the

85 timescales across which rate estimates are time-dependent, and the rate of decay between high

86 pedigree rates and low phylogenetic rate estimates.

87 In particular, quantifying the extent of time-dependent rates is important because it can provide

88 insights into its causes. For example, purifying selection is only expected to operate over

89 relatively short timescales, whereas mutational saturation tends to become a problem at greater

90 time depths (Duchêne et al., 2014; Subramanian et al., 2009). On the other hand, if saturation has

91 only a small impact on rate estimates, there should be an upper limit to the timescale over which

92 the biases can be observed. If these limits and the mode of decay of rate estimates can be

93 identified and incorporated into phylogenetic models, the accuracy of the inference of

94 evolutionary processes will be substantially improved.

95 In this study, we aim to characterize the taxonomic breadth and temporal depth of time-dependent

96 rates by performing a meta-analysis of mitochondrial rates estimated from a wide range of

97 amniote and insect taxa. We investigate how these rate estimates depend on the age of the

98 calibrations used to estimate them, how they vary among taxonomic groups and molecular

99 markers, and whether time-dependent biases persist across a broad temporal scale.

\subsection{Collection of published rate estimates}


102 We performed a meta-analysis of the relationship between evolutionary rate estimates and the

103 timescale over which they are estimated. Our data set comprised published estimates of

104 molecular evolutionary rates, along with the ages of their corresponding calibration points (Table

$105 \mathrm{~S} 1)$. We collected rate estimates for amniotes and insects from recent publications in several

106 leading journals in molecular evolution (Table S1).

107 Because methods used to estimate rates varied widely among studies, we chose the oldest

108 calibration used for rate estimation by each study as a proxy for the timescale over which the rate

109 had been estimated. Therefore, for each rate estimate, 'calibration time' represents (i) the oldest

110 fossil or biogeographical event used as an age constrain on a basal node for the phylogeny; (ii)

111 the oldest ancient DNA sequence used in heterochronous sampling; or (iii) the depth of

112 genealogy in a pedigree study.

\section{$113 \quad 2.2$ Novel rate estimates}

114 In addition to collecting a set of published rate estimates, we obtained thirty novel estimates and

115 re-estimates of rates by analysing available ancient DNA sequences from 16 species (Tables S2).

116 Twelve of these estimates were obtained using ancient DNA as the sole source of calibration. The

117 remaining 18 estimates were obtained from ancient DNA data sets, but with the addition of a

118 single sequence from a sister species and a fossil-based estimate of the divergence time as

119 calibration. Identification of sister species and divergence times was done with reference to

120 published studies (Table S2). One additional rate estimate was obtained using a mitochondrial

121 data set from modern humans and an orangutan, calibrated using fossil evidence of the

122 divergence time between these two species (Table S2). 
123 The best-fitting model of nucleotide substitution was chosen for each data set according to the

124 Bayesian Information Criterion using ModelGenerator 0.85 (Keane et al., 2006). Substitution

125 rates were estimated using the Bayesian phylogenetic software BEAST 1.7.2 (Drummond \&

126 Rambaut, 2007). We used a strict clock calibrated only by the ages of the sequences in the

127 analyses of intraspecific data (Brown \& Yang, 2011; Drummond et al., 2006) and an uncorrelated

128 lognormal relaxed clock when analysing the data sets that included sister species. A uniform prior

129 of 0 to $10^{-4}$ substitutions/site/year was specified for the mean substitution rate, whereas a $1 / \mathrm{x}$ prior

130 was used for the population size in the coalescent prior for the tree. In analyses of data sets that

131 included sister species, an informative prior distribution was specified for the age of the root

132 according to the fossil-based estimate of the divergence time (Table S2). Posterior distributions of

133 parameters were estimated using Markov chain Monte Carlo sampling, with samples drawn every

$13410^{3}$ steps over a total of at least $10^{7}$ steps. Some chains were extended to ensure sufficient

135 sampling and effective sample sizes above 100 for all parameters.

136 For each data set the analysis was repeated using Bayesian skyride model with a default gamma

137 precision prior. The fit of the two population models were compared using Bayes factors

138 calculated in Tracer v1.5 (Rambaut \& Drummond, 2007) and the rate estimate for the model with

139 better fit was used. Median estimates of the strict clock rate for the intraspecific analyses and of

140 the mean rate over the whole inferred phylogeny for the analyses including sister species were

141 taken as the "rate estimates" to be included in all further analyses.

\section{$142 \quad 2.3$ Meta-analysis of the rate estimates}

143 We conducted a meta-analysis of all of the rate estimates (previously published and generated in

144 this study) by plotting log-transformed rate estimates against log-transformed calibration times. 
145 To avoid biasing the regression analysis with multiple estimates for single species, estimates from

146 the various human pedigree studies were pooled together and used as a single data point during

147 meta-analysis (Table S1). For the same reason, we took the average of the human rate estimates

148 calibrated using the Homo-Pan divergence time (Table S1). When a study reported rate estimates

149 from a number of mitochondrial markers for a single species, we took the average of the

150 estimates.

151 We did separate linear regressions for coding and non-coding markers, and for subsets based on

152 taxonomic classes (insects, reptiles, birds, and mammals). We also performed an intraspecific

153 regression on the human data to test for time-dependent rates at the intraspecific level (Table S1).

154 All linear regressions were performed in R (R Development Core Team, 2014).

155 To test whether the strength of time-dependence varied across timescales, we performed

156 regression analyses for subsets of the data, chosen according to calibration times. We used a

157 sliding window with a width of four orders of magnitude $\left(10^{0}-10^{4}\right.$ years, $10^{1}-10^{5}$ years, $\ldots, 10^{5}-$

$15810^{9}$ years). To investigate the extent of the timescale over which time-dependent biases in rate

159 estimation can be observed, we performed regression analyses on subsets of data based on

160 calibrations of $10^{6}-10^{9}, 10^{7}-10^{9}$, and $10^{8}-10^{9}$ years.

\section{$161 \quad 2.4$ Testing for spurious self-correlation}

162 Any correlation between substitution rates and timescales over which they are estimated might be

163 mathematical artifact caused by self-correlation. This phenomenon can occur when a ratio (here,

164 the substitution rate, representing genetic distance divided by time elapsed) is plotted against its

165 denominator (here, time elapsed) and can produce spurious correlations that are highly significant

166 (Kenney, 1982). 
167 For all of our regression analyses, we investigated whether the correlation we detect is solely

168 such a mathematical artifact (Kenney, 1982; Sheets \& Mitchell, 2001) using a randomization test

169 (Jackson \& Somers, 1991). For all the data points in our analysis, we randomized the genetic

170 distances, which we calculated by multiplying substitution rate and calibration time. We

171 recalculated substitution rates using these randomized distances, then performed regression

172 analyses against the corresponding calibration times. This procedure was repeated 10,000 times

173 for each subset of data for which regression analysis was performed in our meta-analysis.

174 We then tested whether the estimate of the regression slope for the original data fell within the

175 distribution of the estimated slopes for the randomized data. In this way we determined whether

176 or not the detected correlations were entirely the product of spurious self-correlation. Deviation

177 from the distribution produced by the randomized data would signify that the observed time-

178 dependence could not be exclusively a mathematical artifact.

179 For all data subsets tested, the randomized data produced an average regression slope of -1 ,

180 which is the value expected when there is spurious self-correlation (Kenney, 1982). Therefore, we

181 performed an additional test by repeating the regression analysis for each subset of data using a

182 null hypothesis of slope $=-1$.

\section{3. Results}

184 Our analyses were based on a data set comprising 239 published, re-estimated, and novel

185 estimates of evolutionary rates in metazoan mitochondrial DNA (Table S1). Rate estimates

186 ranged from $1.78 \times 10^{-10}$ substitutions/site/year (cytochrome $b$ and tRNA genes in woolly

187 mammoth; Rohland et al., 2010) to $2.31 \times 10^{-6}$ substitutions/site/year (the control region in Adélie

188 penguins; Lambert et al., 2002). Calibration ages ranged from 6 years (pedigree study of Adélie 
189 penguins; Millar et al., 2008) to $4 \times 10^{8}$ years (fossil calibration for Bacillus stick insects; Plazzi et 190 al., 2011).

191 To test the relationship between mitochondrial rate estimates and calibration ages, we performed 192 separate linear-regression analyses of coding and non-coding regions. All linear regressions were 193 performed using log-transformed values of both estimated rates and calibration times. We found 194 significant correlations between these two variables in coding markers ( slope $=-0.28, \mathrm{p}=$ $\left.1951.58 \times 10^{-12}, \mathrm{R}^{2}=0.24\right)$ as well as non-coding markers ( 196 (Fig. 1, Table S3).

197 To test whether time-dependent biases were present across the entire timescale that we studied, 198 we performed additional regression analyses for subsets of the data that represented different 199 calibration ages. We found significant non-zero relationships for all time slices containing more 200 than 12 data points (Table 1, Table S4). This includes time depths of $>10^{7}$ years for coding and $201>10^{6}$ years for non-coding markers.

202 We found significant negative relationships between mitochondrial rate estimates and calibration 203 ages in our separate analyses of coding and non-coding markers in mammals, birds, reptiles, and 204 insects (Fig. 2, Table S3). However, there were too few non-coding data points for reptiles (2 data 205 points) and insects ( 0 data points) to allow regression analysis. We also found support for time206 dependent rates in our intraspecific analysis of mitochondrial sequence data from humans (Fig. 207 S1, Table S3), corroborating results previously published for a smaller data set (Henn et al., 208 2009).

209 Each of the significant regressions reported above was significantly different from a 210 corresponding regression that was calculated after randomizing the data and significantly

211 different from a null hypothesis of slope $=-1$, which was the average slope produced by 
212 randomized data (Tables 1, S3 and S4) as expected in the case of spurious self-correlation 213 (Kenney, 1982).

\section{Discussion}

215 Our analyses demonstrate a consistent pattern of time-dependent biases in rate estimates in

216 mitochondrial DNA across metazoan taxa. We observed this pattern across the entire timescale

217 that was analysed, beyond at least $10^{6}$ years in non-coding and $10^{7}$ years in coding markers

218 (Tables 1 and S4). This is in contrast with previous estimates of the temporal depth of time-

219 dependence, which has been variously estimated at a few hundred years (Richards M. in Gibbons,

220 1998), around 50 kyr (Henn et al., 2009), 200 kyr (Burridge et al., 2008), or 1-2 Myr (Ho et al.,

221 2005; Papadopoulou et al., 2010). However, our finding of a prolonged decay in molecular rate

222 estimates is consistent with recent evidence from a large-scale analysis of substitution rates in

223 viruses, which revealed a time-dependent bias in rate estimates across a temporal scale spanning

22410 orders of magnitude (Duchêne et al., 2014). Together, these results have considerable

225 implications for studies of divergence times.

226 Our finding of a prolonged time-dependent bias in rate estimates sheds some light on the causes

227 of time-dependent biases in rate estimation. Specifically, the temporal breadth of the relationship

228 between rate and calibration time suggests that more than one factor is responsible. The effects of

229 purifying selection decline over time and thus are not expected to be detectable over long

230 timescales (Nei, 1971; Phillips, 2009), even though the lack of recombination in mitochondrial

231 DNA can potentially extend the life of deleterious mutations through genetic hitchhiking (Ballard

$232 \&$ Kreitman, 1995; Birky \& Walsh, 1988). In turn, mutational saturation is expected to increase

233 over time and, if the available nucleotide substitution models do not correct for multiple hits 
234 sufficiently, the rates estimated over longer timescales will be more severely underestimated.

235 Underestimation of the number of substitutions across longer timeframes can lead to time-

236 dependent patterns in other evolutionary parameters, including the ratio of nonsynonymous to

237 synonymous substitutions (dos Reis \& Yang, 2013) and the ratio of transitions to transversions

238 (Duchêne et al., In press). Therefore, we propose that purifying selection and mutational

239 saturation are the main drivers of these biases. This is consistent with the patterns found in

240 sequence data from viruses (Duchêne et al., 2014).

241 Signs of a time-dependent bias in rate estimation persisting for more than $10 \mathrm{Myr}$ and in a wide

242 range of taxa also allow us to challenge some other hypotheses about the causes of time-

243 dependent rates. Population fluctuations and other demographic factors (Balloux \& Lehmann,

244 2012; Henn et al., 2009), which vary among species, cannot fully explain the patterns that we

245 have observed across a diverse range of taxa. Given the temporal depth of time-dependent biases

246 in rate estimation, calibration errors are unlikely to represent a sufficient explanation (Ho et al.,

247 2011; Peterson \& Masel, 2009). Our data-randomization analyses suggest that the time-dependent

248 pattern of rate estimates is unlikely to be solely a mathematical artefact. Even if this were the

249 case, however, accounting for time-dependent biases in rate estimates would still be necessary

250 when estimating evolutionary timescales using the molecular clock.

251 Our results also support a gradual decay from high pedigree to low phylogenetic rate estimates

252 through time. Intermediate rate estimates, such as those obtained from ancient DNA studies, have

253 previously been attributed to various methodological biases (Debruyne \& Poinar, 2009; Emerson

254 \& Hickerson, In press; Navascues \& Emerson, 2009). However, our results do not indicate that

255 rate estimates based on ancient DNA are anomalous. Additionally, estimates of rates calibrated

256 using biogeographic events in the late Pleistocene and Holocene are comparable to those based 
257 on ancient DNA data of similar age. This can be seen, for example, in our intraspecific analysis

258 of rate estimates from humans (Fig. S1, Table S1).

259 One of the disadvantages of our meta-analysis is the paucity of mitochondrial rate estimates

260 based on young calibrations for coding markers and for insects. Although the uneven

261 representation of taxonomic classes on different timescales might affect the regression analysis

262 that includes all data points, our analyses of class-specific subsets of data yielded significant

263 evidence of time-dependent trends. One other potential source of bias in the meta-analysis is that

264 studies of evolutionary processes on short timescales tend to focus on fast-evolving taxa, whereas

265 studies of longer timescales have a greater focus on slowly evolving organisms (Sheets \&

266 Mitchell, 2001). This, however, can be rejected as a major cause for the biases based on the

267 observed intraspecific time-dependent variation of estimated rates, which was shown previously

268 (Duchêne et al., 2014; Henn et al., 2009) and in this study (Fig. S1, Table S3).

269 Our results confirm that considerable caution needs to be exercised in molecular studies of

270 evolutionary timescales. The need to correct for time-dependent biases in rate estimates has been

271 pointed out previously (Ho et al., 2005). As the variability of regression slopes in our study

272 shows, however, there is no universal function that can be applied across taxa and markers.

273 Although a few studies have proposed corrections for specific taxa (Cutter, 2008; Gignoux et al.,

274 2011; Obbard et al., 2012; Soares et al., 2013; Soares et al., 2009), they focused on the effects of

275 purifying selection. Additionally, it is not clear to what extent they can be extrapolated to other

276 taxa. Ultimately, it might be best to only use calibrations that are reasonably close in time to the

277 evolutionary events of interest (Ho \& Larson, 2006).

278 In view of the prevalence of time-dependent biases in estimates of mitochondrial rates, neutrally

279 evolving nuclear loci might be a preferable alternative to the mitochondrial genome for

280 estimating evolutionary timescales. However, there has been compelling evidence of time- 
281 dependent biases in estimates of nuclear rates in some organisms, including Caenorhabditis

282 elegans (Denver et al., 2004) and Drosophila melanogaster (Haag-Liautard et al., 2007). In

283 contrast, studies of human nuclear genomes have yielded lower estimates of rates from pedigrees

284 than from fossil-calibrated comparisons with chimpanzees (Roach et al., 2010; Xue et al., 2009).

285 This represents a prominent anomaly against the broad time-dependent biases seen in other

286 estimates of evolutionary rates, and it remains to be seen whether the results from human

287 genomes are representative of a broader trend among nuclear genomes (Scally \& Durbin, 2012).

\section{Acknowledgements}

289 The authors would like to thank William Amos, Brian Golding, and Mario dos Reis for their 290 helpful comments on the paper, and Sebastián Duchêne for help with data analyses and for 291 stimulating discussions.

292 References

293 Ballard JW, and Kreitman M. 1995. Is mitochondrial DNA a strictly neutral marker? Trends in $294 \quad$ Ecology and Evolution 10:485-488.

295 Balloux F, and Lehmann L. 2012. Substitution rates at neutral genes depend on population size 296 under fluctuating demography and overlapping generations. Evolution 66:605-611.

297 Benton MJ, and Ayala FJ. 2003. Dating the tree of life. Science 300:1698-1700.

298

299

300

Birky CW, Jr, and Walsh JB. 1988. Effects of linkage on rates of molecular evolution. Proceedings of the National Academy of Sciences of the United States of America 85:6414-6418. 
301

302

303

304

305

306

307

308

309

310

311

312

313

314

315

316

317

318

319

320

321

322

323

324

325

326

327

328

329

330

331

332

333

334

335

336
Bromham L. 2009. Why do species vary in their rate of molecular evolution? Biology Letters 5:401-404.

Brower AVZ. 1994. Rapid morphological radiation and convergence among races of the butterfly Heliconius erato inferred from patterns of mitochondrial DNA evolution. Proceedings of the National Academy of Sciences of the United States of America 91:6491-6495.

Brown RP, and Yang Z. 2011. Rate variation and estimation of divergence times using strict and relaxed clocks. BMC Evolutionary Biology 11:271.

Brown WM, George M, and Wilson AC. 1979. Rapid evolution of animal mitochondrial DNA. Proceedings of the National Academy of Sciences of the United States of America 76:1967-1971.

Burridge CP, Craw D, Fletcher D, and Waters JM. 2008. Geological dates and molecular rates: fish DNA sheds light on time dependency. Molecular Biology and Evolution 25:624-633.

Crandall ED, Sbrocco EJ, Deboer TS, Barber PH, and Carpenter KE. 2012. Expansion dating: calibrating molecular clocks in marine species from expansions onto the Sunda Shelf following the Last Glacial Maximum. Molecular Biology and Evolution 29:707-719.

Cutter AD. 2008. Divergence times in Caenorhabditis and Drosophila inferred from direct estimates of the neutral mutation rate. Molecular Biology and Evolution 25:778-786.

Debruyne R, and Poinar HN. 2009. Time dependency of molecular rates in ancient DNA data sets, a sampling artifact? Systematic Biology 58:348-360.

Denver DR, Morris K, Lynch M, and Thomas WK. 2004. High mutation rate and predominance of insertions in the Caenorhabditis elegans nuclear genome. Nature 430:679-682.

dos Reis M, and Yang Z. 2013. Why do more divergent sequences produce smaller nonsynonymous/synonymous rate ratios in pairwise sequence comparisons? Genetics 195:195-204.

Drummond AJ, Ho SYW, Phillips MJ, and Rambaut A. 2006. Relaxed phylogenetics and dating with confidence. PLOS Biology 4:e88.

Drummond AJ, Pybus OG, Rambaut A, Forsberg R, and Rodrigo AG. 2003. Measurably evolving populations. Trends in Ecology and Evolution 18:481-488.

Drummond AJ, and Rambaut A. 2007. BEAST: Bayesian evolutionary analysis by sampling trees. BMC Evolutionary Biology 7:214.

Duchêne S, Ho SYW, and Holmes EC. In press. Declining transition/transversion ratios through time reveal limitations to the accuracy of nucleotide substitution models. $B M C$ Evolutionary Biology.

Duchêne S, Holmes EC, and Ho SYW. 2014. Analyses of evolutionary dynamics in viruses are hindered by a time-dependent bias in rate estimates. Proceedings of the Royal Society $B$ - Biological Sciences 281:20140732. 
Emerson BC. 2007. Alarm bells for the molecular clock? No support for Ho et al.'s model of time-dependent molecular rate estimates. Systematic Biology 56:337-345.

Emerson BC, and Hickerson MJ. In press. Lack of support for the time-dependent molecular evolution hypothesis. Molecular Ecology.

Endicott P, Ho SYW, Metspalu M, and Stringer C. 2009. Evaluating the mitochondrial timescale of human evolution. Trends in Ecology and Evolution 24:515-521.

Fleischer RC, McIntosh CE, and Tarr CL. 1998. Evolution on a volcanic conveyor belt: using phylogeographic reconstructions and K-Ar-based ages of the Hawaiian Islands to estimate molecular evolutionary rates. Molecular Ecology 7:533-545.

García-Moreno J, Cortés N, Garcia-Deras GM, and Hernández-Baños BE. 2006. Local origin and diversification among Lampornis hummingbirds: a Mesoamerican taxon. Molecular Phylogenetics and Evolution 38:488-498.

Genner MJ, Seehausen O, Lunt DH, Joyce DA, Shaw PW, Carvalho GR, and Turner GF. 2007. Age of cichlids: new dates for ancient lake fish radiations. Molecular Biology and Evolution 24:1269-1282.

Gibbons A. 1998. Calibrating the mitochondrial clock. Science 279:28-29.

Gignoux CR, Henn BM, and Mountain JL. 2011. Rapid, global demographic expansions after the origins of agriculture. Proceedings of the National Academy of Sciences of the United States of America 108:6044-6049.

Gratton P, Konopiński MK, and Sbordoni V. 2008. Pleistocene evolutionary history of the Clouded Apollo (Parnassius mnemosyne): genetic signatures of climate cycles and a 'time-dependent' mitochondrial substitution rate. Molecular Ecology 17:4248-4262.

Haag-Liautard C, Dorris M, Maside X, Macaskill S, Halligan DL, Charlesworth B, and Keightley PD. 2007. Direct estimation of per nucleotide and genomic deleterious mutation rates in Drosophila. Nature 445:82-85.

Henn BM, Gignoux CR, Feldman MW, and Mountain JL. 2009. Characterizing the time dependency of human mitochondrial DNA mutation rate estimates. Molecular Biology and Evolution 26:217-230.

Hipsley CA, and Müller J. 2014. Beyond fossil calibrations: realities of molecular clock practices in evolutionary biology. Frontiers in Genetics 5.

Ho SYW. 2014. The changing face of the molecular evolutionary clock. Trends in Ecology and Evolution 29:496-503.

Ho SYW, and Duchêne S. 2014. Molecular-clock methods for estimating evolutionary rates and timescales. Molecular Ecology 23:5947-5965.

Ho SYW, Kolokotronis SO, and Allaby RG. 2007a. Elevated substitution rates estimated from ancient DNA sequences. Biology Letters 3:702-705. 
Ho SYW, Lanfear R, Bromham L, Phillips MJ, Soubrier J, Rodrigo AG, and Cooper A. 2011. Time-dependent rates of molecular evolution. Molecular Ecology 20:3087-3101.

Ho SYW, and Larson G. 2006. Molecular clocks: when times are a-changin'. Trends in Genetics 22:79-83.

Ho SYW, and Lo N. 2013. The insect molecular clock. Australian Journal of Entomology 52:101-105.

Ho SYW, Phillips MJ, Cooper A, and Drummond AJ. 2005. Time dependency of molecular rate estimates and systematic overestimation of recent divergence times. Molecular Biology and Evolution 22:1561-1568.

Ho SYW, Saarma U, Barnett R, Haile J, and Shapiro B. 2008. The effect of inappropriate calibration: three case studies in molecular ecology. PLOS ONE 3:e1615.

Ho SYW, Shapiro B, Phillips MJ, Cooper A, and Drummond AJ. 2007b. Evidence for time dependency of molecular rate estimates. Systematic Biology 56:515-522.

Howell N, Smejkal CB, Mackey DA, Chinnery PF, Turnbull DM, and Herrnstadt C. 2003. The pedigree rate of sequence divergence in the human mitochondrial genome: there is a difference between phylogenetic and pedigree rates. American Journal of Human Genetics 72:659-670.

Jackson DA, and Somers KM. 1991. The spectre of spurious correlations. Oecologia 86:147-151.

Jetz W, Thomas GH, Joy JB, Hartmann K, and Mooers AO. 2012. The global diversity of birds in space and time. Nature 491:444-448.

Keane TM, Creevey CJ, Pentony MM, Naughton TJ, and McLnerney JO. 2006. Assessment of methods for amino acid matrix selection and their use on empirical data shows that ad hoc assumptions for choice of matrix are not justified. BMC Evolutionary Biology 6:29.

Kenney BC. 1982. Beware of spurious self-correlations. Water Resources Research 18:10411048.

Kumar S. 2005. Molecular clocks: four decades of evolution. Nature Reviews Genetics 6:654662.

Lambert DM, Ritchie PA, Millar CD, Holland B, Drummond AJ, and Baroni C. 2002. Rates of evolution in ancient DNA from Adélie penguins. Science 295:2270-2273.

Lorenzen ED, Nogues-Bravo D, Orlando L, Weinstock J, Binladen J, Marske KA, Ugan A, Borregaard MK, Gilbert MTP, Nielsen R, Ho SYW, Goebel T, Graf KE, Byers D, Stenderup JT, Rasmussen M, Campos PF, Leonard JA, Koepfli KP, Froese D, Zazula G, Stafford TW, Jr., Aaris-Sorensen K, Batra P, Haywood AM, Singarayer JS, Valdes PJ, Boeskorov G, Burns JA, Davydov SP, Haile J, Jenkins DL, Kosintsev P, Kuznetsova T, Lai X, Martin LD, McDonald HG, Mol D, Meldgaard M, Munch K, Stephan E, Sablin M, Sommer RS, Sipko T, Scott E, Suchard MA, Tikhonov A, Willerslev R, Wayne RK, Cooper A, Hofreiter M, Sher A, Shapiro B, Rahbek C, and Willerslev E. 2011. Species- 
412

413

414

415

416

417

418

419

420

421

422

423

424

425

426

427

428

429

430

431

432

433

434

435

436

437

438

439

440

441

442

443

444 445

specific responses of Late Quaternary megafauna to climate and humans. Nature 479:359-364.

Macey JR, Schulte JA, Ananjeva NB, Larson A, Rastegar-Pouyani N, Shammakov SM, and Papenfuss TJ. 1998. Phylogenetic relationships among agamid lizards of the Laudakia caucasia species group: testing hypotheses of biogeographic fragmentation and an area cladogram for the Iranian Plateau. Molecular Phylogenetics and Evolution 10:118-131.

Millar CD, Dodd A, Anderson J, Gibb GC, Ritchie PA, Baroni C, Woodhams MD, Hendy MD, and Lambert DM. 2008. Mutation and evolutionary rates in Adélie penguins from the Antarctic. PLOS Genetics 4:e1000209.

Molak M, Lorenzen ED, Shapiro B, and Ho SYW. 2013. Phylogenetic estimation of timescales using ancient DNA: the effects of temporal sampling scheme and uncertainty in sample ages. Molecular Biology and Evolution 30:253-262.

Navascues M, and Emerson BC. 2009. Elevated substitution rate estimates from ancient DNA: model violation and bias of Bayesian methods. Molecular Ecology 18:4390-4397.

Nei M. 1971. Extinction time of deleterious mutant genes in large populations. Theoretical Population Biology 2:419-425.

Obbard DJ, Maclennan J, Kim KW, Rambaut A, O'Grady PM, and Jiggins FM. 2012. Estimating divergence dates and substitution rates in the Drosophila phylogeny. Molecular Biology and Evolution 29:3459-3473.

Papadopoulou A, Anastasiou I, and Vogler AP. 2010. Revisiting the insect mitochondrial molecular clock: the mid-Aegean trench calibration. Molecular Biology and Evolution 27:1659-1672.

Peterson GI, and Masel J. 2009. Quantitative prediction of molecular clock and $K_{a} / K_{s}$ at short timescales. Molecular Biology and Evolution 26:2595-2603.

Phillips MJ. 2009. Branch-length estimation bias misleads molecular dating for a vertebrate mitochondrial phylogeny. Gene 441:132-140.

Plazzi F, Ricci A, and Passamonti M. 2011. The mitochondrial genome of Bacillus stick insects (Phasmatodea) and the phylogeny of orthopteroid insects. Molecular Phylogenetics and Evolution 58:304-316.

R Development Core Team. 2014. R: a language and environment for statistical computing. Vienna, Austria, URL http://www.R-project.org/: R foundation for statistical computing.

Rambaut A. 2000. Estimating the rate of molecular evolution: incorporating noncontemporaneous sequences into maximum likelihood phylogenies. Bioinformatics 16:395-399.

Rambaut A, and Drummond AJ. 2007. Tracer v1.5, Available from http://beast.bio.ed.ac.uk/Tracer 
464 465

466

467

468

469

470 471

472

473

474

475

476 477

478 479

480 481

Reed DL, Light JE, Allen JM, and Kirchman JJ. 2007. Pair of lice lost or parasites regained: the evolutionary history of anthropoid primate lice. BMC Biology 5:7.

Rieux A, Eriksson A, Li M, Sobkowiak B, Weinert LA, Warmuth V, Ruiz-Linares A, Manica A, and Balloux F. 2014. Improved calibration of the human mitochondrial clock using ancient genomes. Molecular Biology and Evolution 31:2780-2792.

Roach JC, Glusman G, Smit AFA, Huff CD, Hubley R, Shannon PT, Rowen L, Pant KP, Goodman N, Bamshad M, Shendure J, Drmanac R, Jorde LB, Hood L, and Galas DJ. 2010. Analysis of genetic inheritance in a family quartet by whole-genome sequencing. Science 328:636-639.

Rohland N, Reich D, Mallick S, Meyer M, Green RE, Georgiadis NJ, Roca AL, and Hofreiter M. 2010. Genomic DNA sequences from mastodon and woolly mammoth reveal deep speciation of forest and savanna elephants. PLOS Biology 8:e1000564.

Scally A, and Durbin R. 2012. Revising the human mutation rate: implications for understanding human evolution. Nature Reviews Genetics 13:745-753.

Sheets HD, and Mitchell CE. 2001. Uncorrelated change produces the apparent dependence of evolutionary rate on interval. Paleobiology 27:429-445.

Shields GF, and Wilson AC. 1987. Calibration of mitochondrial DNA evolution in geese. Journal of Molecular Evolution 24:212-217.

Soares P, Abrantes D, Rito T, Thomson N, Radivojac P, Li B, Macaulay V, Samuels DC, and Pereira L. 2013. Evaluating purifying selection in the mitochondrial DNA of various mammalian species. PLOS ONE 8:e58993.

Soares P, Ermini L, Thomson N, Mormina M, Rito T, Rohl A, Salas A, Oppenheimer S, Macaulay V, and Richards MB. 2009. Correcting for purifying selection: an improved human mitochondrial molecular clock. American Journal of Human Genetics 84:740-759.

Soubrier J, Steel M, Lee MSY, Sarkissian CD, Guindon S, Ho SYW, and Cooper A. 2012. The influence of rate heterogeneity among sites on the time dependence of molecular rates. Molecular Biology and Evolution 29:3345-3358.

Subramanian S, Denver DR, Millar CD, Heupink T, Aschrafi A, Emslie SD, Baroni C, and Lambert DM. 2009. High mitogenomic evolutionary rates and time dependency. Trends in Genetics 25:482-486.

Weir JT, and Schluter D. 2008. Calibrating the avian molecular clock. Molecular Ecology 17:2321-2328.

Woodhams M. 2006. Can deleterious mutations explain the time dependency of molecular rate estimates? Molecular Biology and Evolution 23:2271-2273.

Xue YL, Wang QJ, Long Q, Ng BL, Swerdlow H, Burton J, Skuce C, Taylor R, Abdellah Z, Zhao YL, Asan, MacArthur DG, Quail MA, Carter NP, Yang HM, and Tyler-Smith C. 2009. 
Human Y chromosome base-substitution mutation rate measured by direct sequencing in

483 a deep-rooting pedigree. Current Biology 19:1453-1457.

484 Zuckerkandl E, and Pauling L. 1962. Molecular disease, evolution and genic heterogeneity. In: 485 Kasha M, and Pullman B, eds. Horizons in Biochemistry. New York, USA: Academic 486

Press, 189-225. 


\section{1}

Figure 1

Linear regressions of log-transformed rate estimates from mitochondrial markers in a range of metazoan taxa against the log-transformed calibration times that were used to estimate the rates. Separate analyses were performed for coding (A) and non-coding (B) markers.

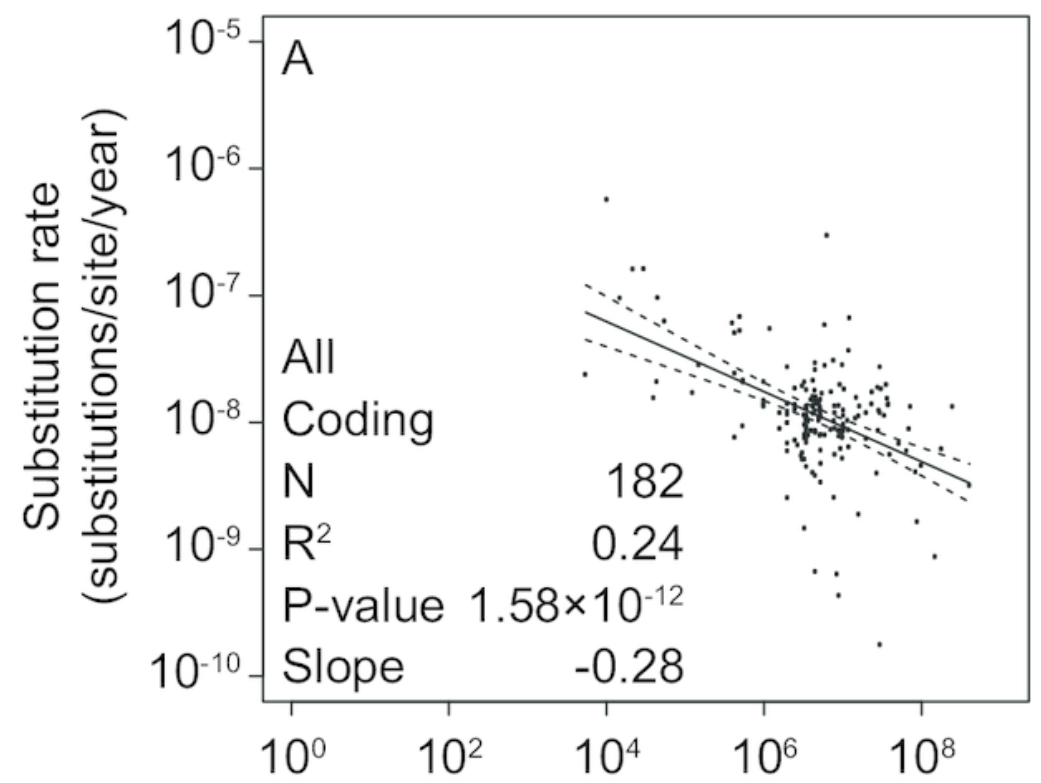

Calibration time (years)

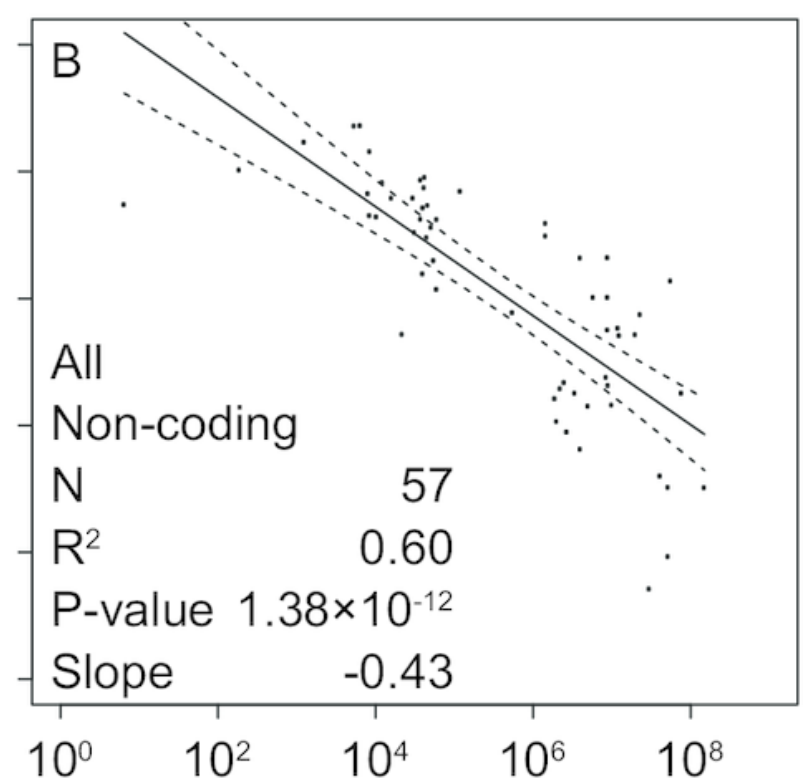

Calibration time (years) 


\section{Table $\mathbf{1}$ (on next page)}

Table 1

Linear-regression analysis of data points from different time slices using a sliding window with a width of four orders of magnitude, or with a shrinking window for the oldest calibrations. Significant regression results $(p \leq 0.05)$ are shown in bold. A more detailed version of this table is available as supplementary material (Table $\mathrm{S} 4$ ). 


\begin{tabular}{|c|c|c|c|c|c|c|c|c|c|c|}
\hline & \multicolumn{9}{|c|}{ calibration ages } \\
\hline & & $\begin{array}{r}10^{0}-10^{4} \\
\text { years }\end{array}$ & $\begin{array}{r}10^{1}-10^{5} \\
\text { years }\end{array}$ & $\begin{array}{r}10^{2}-10^{6} \\
\text { years }\end{array}$ & $\begin{array}{r}10^{3}-10^{7} \\
\text { years }\end{array}$ & $\begin{array}{r}10^{4}-10^{8} \\
\text { years }\end{array}$ & $\begin{array}{r}10^{5}-10^{9} \\
\text { years }\end{array}$ & $\begin{array}{r}10^{6}-10^{9} \\
\text { years }\end{array}$ & $\begin{array}{r}10^{7}-10^{9} \\
\text { years }\end{array}$ & $\begin{array}{r}10^{8}-10^{9} \\
\text { years }\end{array}$ \\
\hline \multirow{5}{*}{ 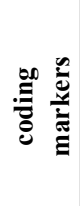 } & $\mathbf{N}$ & 1 & 9 & 20 & 132 & 176 & 173 & 162 & 50 & 5 \\
\hline & $\mathbf{R}^{2}$ & 0 & 0.05 & 0.24 & 0.31 & 0.23 & 0.09 & 0.04 & 0.11 & 0.04 \\
\hline & P-value & $\mathrm{n} / \mathrm{a}$ & $5.67 \times 10^{-1}$ & $2.67 \times 10^{-2}$ & $3.43 \times 10^{-12}$ & $1.48 \times 10^{-11}$ & $4.79 \times 10^{-5}$ & $1.26 \times 10^{-2}$ & $1.76 \times 10^{-2}$ & $7.6 \times 10^{-1}$ \\
\hline & Slope & $\mathrm{n} / \mathrm{a}$ & -0.33 & -0.34 & -0.37 & -0.29 & -0.20 & -0.15 & -0.34 & 0.36 \\
\hline & Non-SSC ${ }^{\text {a }}$ & $\mathrm{n} / \mathrm{a}$ & - & $* * *$ & $* * *$ & $* * *$ & $* * *$ & $* * *$ & $* * *$ & - \\
\hline \multirow{5}{*}{ 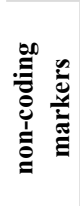 } & $\mathbf{N}$ & 8 & 25 & 27 & 43 & 48 & 31 & 29 & 12 & 1 \\
\hline & $\mathbf{R}^{2}$ & 0.12 & 0.27 & 0.33 & 0.62 & 0.55 & 0.27 & 0.17 & 0.16 & 0 \\
\hline & P-value & $4.09 \times 10^{-1}$ & $7.41 \times 10^{-3}$ & $1.80 \times 10^{-3}$ & $4.47 \times 10^{-10}$ & $1.41 \times 10^{-9}$ & $2.58 \times 10^{-3}$ & $2.55 \times 10^{-2}$ & $2.00 \times 10^{-1}$ & $\mathrm{n} / \mathrm{a}$ \\
\hline & Slope & 0.08 & -0.34 & -0.36 & -0.48 & -0.49 & -0.58 & -0.53 & -0.89 & $\mathrm{n} / \mathrm{a}$ \\
\hline & Non-SSC ${ }^{\text {a }}$ & $* *$ & $* * *$ & $* * *$ & $* * *$ & $* * *$ & $*$ & * & - & $\mathrm{n} / \mathrm{a}$ \\
\hline
\end{tabular}

2 aResults of tests against spurious self-correlation (non-SSC). Higher $\mathrm{p}$-value from the two tests is shown: * $\mathrm{p}<0.05$, ** $3 \mathrm{p}<0.005, * * * \mathrm{p}<0.0005$. 


\section{2}

Figure 2

Linear regressions of log-transformed rate estimates against log-transformed calibration times used for their estimation for different taxonomic groups (insects [A], reptiles [D], birds $[B, E]$, and mammals $[C, F]$ ) and mitochondrial marker types (coding $[A-D]$ and non-coding $[E$, F]). There were insufficient data for regression analyses of non-coding markers in reptiles and insects.
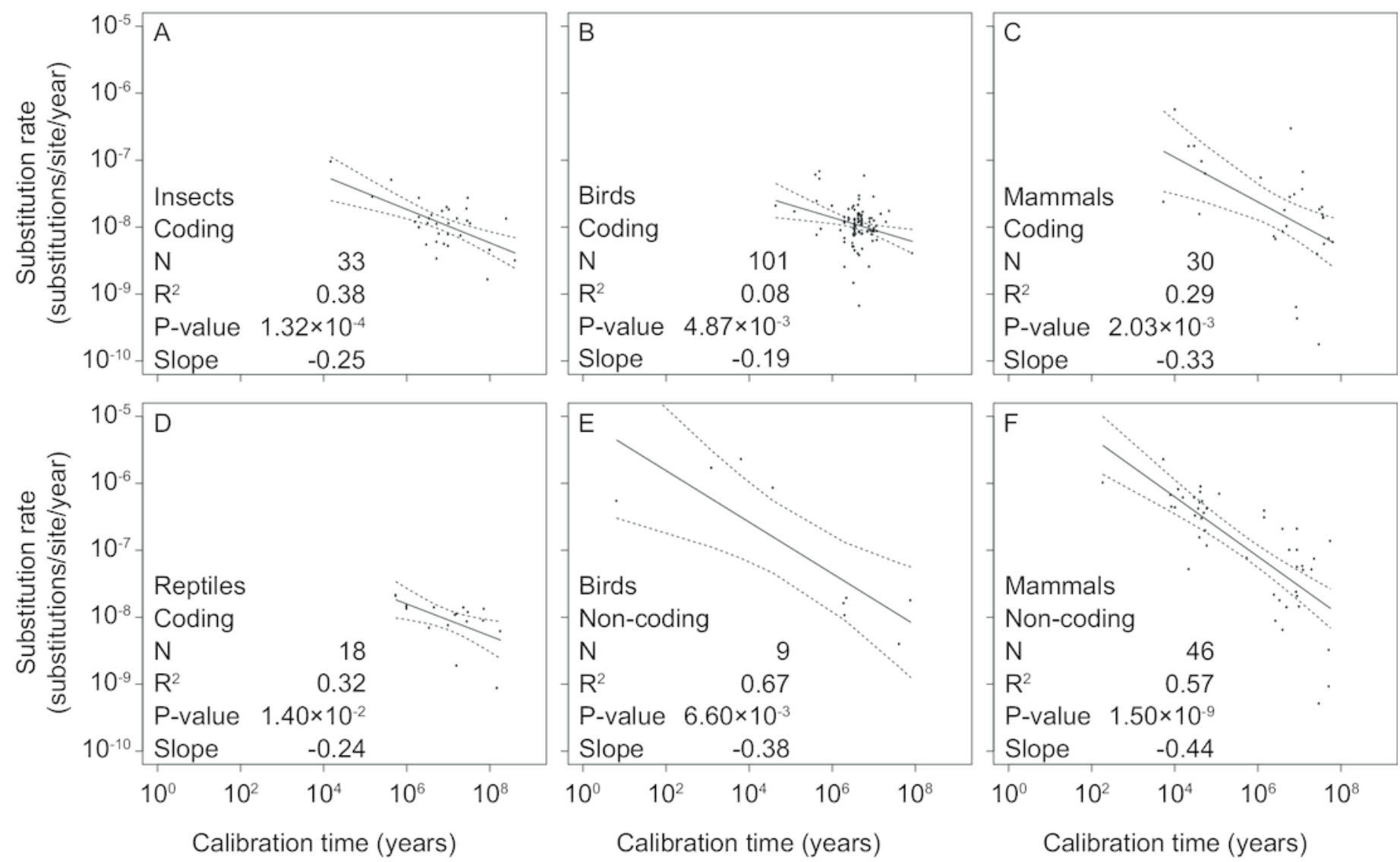